\title{
High beta lasing in micropillar cavities with adiabatic layer design
}

Lermer, M.; Gregersen, Niels; Lorke, M.; Schild, E.; Gold, P.; Mørk, Jesper; Schneider, C.; Forchel, A.; Reitzenstein, S.; Höfling, S.

Total number of authors:

11

Published in:

Applied Physics Letters

Link to article, DOI:

$10.1063 / 1.4791563$

Publication date:

2013

Document Version

Publisher's PDF, also known as Version of record

Link back to DTU Orbit

Citation $(A P A)$ :

Lermer, M., Gregersen, N., Lorke, M., Schild, E., Gold, P., Mørk, J., Schneider, C., Forchel, A., Reitzenstein, S., Höfling, S., \& Kamp, M. (2013). High beta lasing in micropillar cavities with adiabatic layer design. Applied Physics Letters, 102(5), 052114. https://doi.org/10.1063/1.4791563

\section{General rights}

Copyright and moral rights for the publications made accessible in the public portal are retained by the authors and/or other copyright owners and it is a condition of accessing publications that users recognise and abide by the legal requirements associated with these rights.

- Users may download and print one copy of any publication from the public portal for the purpose of private study or research.

- You may not further distribute the material or use it for any profit-making activity or commercial gain

- You may freely distribute the URL identifying the publication in the public portal 


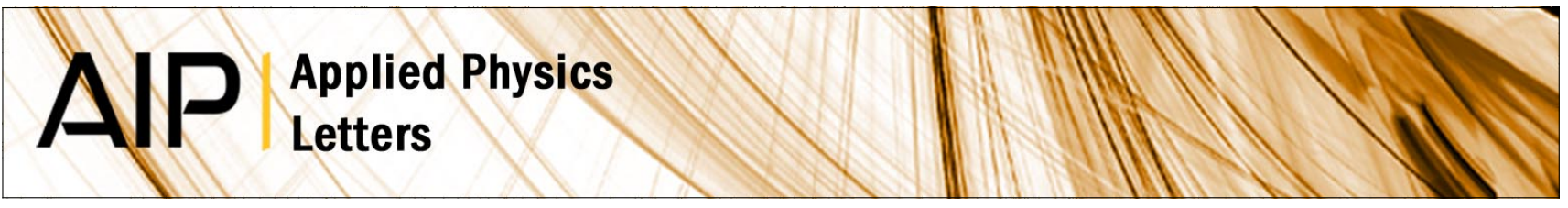

\section{High beta lasing in micropillar cavities with adiabatic layer design}

M. Lermer, N. Gregersen, M. Lorke, E. Schild, P. Gold et al.

Citation: Appl. Phys. Lett. 102, 052114 (2013); doi: 10.1063/1.4791563

View online: http://dx.doi.org/10.1063/1.4791563

View Table of Contents: http://apl.aip.org/resource/1/APPLAB/v102/i5

Published by the American Institute of Physics.

\section{Related Articles}

Portable optical-resolution photoacoustic microscopy with a pulsed laser diode excitation Appl. Phys. Lett. 102, 053704 (2013)

Electro-optical and lasing properties of hybrid quantum dot/quantum well material system for reconfigurable photonic devices

Appl. Phys. Lett. 102, 053110 (2013)

Laser emissions from one-dimensional photonic crystal rings on silicon-dioxide

Appl. Phys. Lett. 102, 051103 (2013)

High-brightness tapered quantum cascade lasers

Appl. Phys. Lett. 102, 053503 (2013)

Suppression of spatial hole burning and pulse stabilization for actively modelocked quantum cascade lasers using quantum coherent absorption

J. Appl. Phys. 113, 053106 (2013)

\section{Additional information on Appl. Phys. Lett.}

Journal Homepage: http://apl.aip.org/

Journal Information: http://apl.aip.org/about/about_the_journal

Top downloads: http://apl.aip.org/features/most_downloaded

Information for Authors: http://apl.aip.org/authors

\section{ADVERTISEMENT}

\section{AIP $\begin{aligned} & \text { Applied Physics } \\ & \text { Letters }\end{aligned}$}

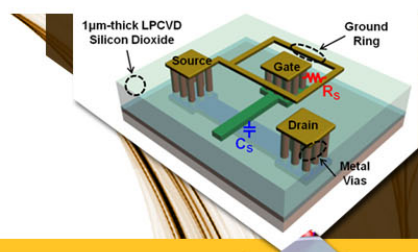

SURFACES AND INTERFACES

Focusing on physical, chemical, biological structural, optical, magnetic and electrical properties of surfaces and interfaces, and more..

\section{EXPLORE WHAT'S NEW IN APL}

SUBMIT YOUR PAPER NOW!
ENERCY CONVERSION AND STORACE 


\title{
High beta lasing in micropillar cavities with adiabatic layer design
}

\author{
M. Lermer, ${ }^{1}$ N. Gregersen, ${ }^{2}$ M. Lorke,${ }^{3}$ E. Schild, ${ }^{1}$ P. Gold, ${ }^{1}$ J. Mørk,${ }^{2}$ C. Schneider, ${ }^{1}$ \\ A. Forchel, ${ }^{1}$ S. Reitzenstein, ${ }^{1, a)}$ S. Höfling, ${ }^{1}$ and M. Kamp ${ }^{1}$ \\ ${ }^{1}$ Technische Physik, Wilhelm Conrad Röntgen Research Center for Complex Material Systems, \\ Universität Würzburg, Am Hubland, D-97074 Würzburg, Germany \\ ${ }^{2}$ DTU Fotonik, Department of Photonics Engineering, Technical University of Denmark, Building 343 , \\ DK-2800 Kongens Lyngby, Denmark \\ ${ }^{3}$ Bremen Center for Computational Materials Science, University of Bremen, Germany
}

(Received 19 December 2012; accepted 28 January 2013; published online 7 February 2013)

\begin{abstract}
We report on lasing in optically pumped adiabatic micropillar cavities, based on the AlAs/GaAs material system. A detailed study of the threshold pump power and the spontaneous emission $\beta$ factor in the lasing regime for different diameters $d_{c}$ is presented. We demonstrate a reduction of the threshold pump power by over 2 orders of magnitude from $d_{c}=2.25 \mu \mathrm{m}$ down to $0.95 \mu \mathrm{m}$. Lasing with $\beta$ factors exceeding 0.5 shows that adiabatic micropillars are operating deeply in the cavity quantum electrodynamics regime. (c) 2013 American Institute of Physics.

[http://dx.doi.org/10.1063/1.4791563]
\end{abstract}

Semiconductor quantum dot (QD)-microcavity systems are highly interesting for the study of fundamental lightmatter interaction and the development of innovative quantum devices. For instance, QD-micropillar (MP) cavities providing three dimensional photon confinement ${ }^{1,2}$ have been utilized as a model system for the demonstration of cavity quantum electrodynamics (cQED) effects such as Purcell-enhancement, ${ }^{3}$ strong light-matter coupling, ${ }^{4}$ and their exploitation for the efficient emission of single ${ }^{5-7}$ and indistinguishable ${ }^{8}$ photons as well as entangled photon pairs. ${ }^{9}$ For a notable enhancement of the spontaneous emission (SE) of single QDs, it is crucial to confine light in microresonators providing high quality $(Q)$ factors and low mode volumes $\left(V_{\text {mode }}\right)$. Indeed, the figure of merit for the weak coupling regime, the so called Purcell factor $F_{P}$ for a single monochromatic emitter, is proportional to $Q / V_{\text {mode }}{ }^{10}$ Remarkably high $Q$ factors have been demonstrated for MPs with diameters $\left(d_{c}\right)$ in the range of a few micrometers, leading to expected $F_{P}$ values of ca. $50-70$ in MPs with $d_{c} \sim 2 \mu \mathrm{m} .^{4,11,12}$ Because of the efficient coupling of SE into the fundamental mode, lasing operation, both under optical and electrical pumping, has been demonstrated in MP cavities containing less than 100 QDs in the active layer and with $\beta$ factors of $0.2-0.3^{13,14}$ and even single QD lasing effects have been demonstrated. ${ }^{15-17}$ Aiming for the realization of thresholdless lasing operation ${ }^{18}$ in MP cavities, further optimization has to be accomplished. By implementing a recently developed bandgap tapering technique for MP cavities, mode matching between the fundamental Bloch mode and the DBR Bloch modes is strongly improved and high $Q$ factors exceeding 10.000 have been demonstrated for submicron diameter pillars. ${ }^{19}$ Together with small mode volumes between 2 and 3 $\left(\lambda_{c} / n\right)^{3}$ in this diameter regime, nominal Purcell factors of several hundreds can be estimated, paving the way for low threshold and high $\beta$ lasing operation. In this letter, we investigate the influence of diameter variations on the lasing per-

\footnotetext{
${ }^{a}$ Present address: Institut für Festkörperphysik, Technische Universität Berlin, Hardenbergstraße 36, D-10623 Berlin, Germany.
}

formance of adiabatic MP cavities and demonstrate low threshold $(3 \mu \mathrm{W})$ and high $\beta(>0.5)$ lasing for $d_{c}=0.95 \mu \mathrm{m}$.

The MPs under investigation have been grown by molecular beam epitaxy and processed using electron-beam lithography and plasma reactive ion etching. By means of ion-beam milling, a MP with $d_{c}=0.8 \mu \mathrm{m}$ has been cut vertically, offering a cross sectional view by means of scanning electron microscope (SEM) imaging (inset in Fig. 1(a)). Slight fabrication induced imperfections, namely, the underetching of AlAs layers and a glass coating of the MPs can be seen. ${ }^{11,20} \mathrm{~A}$ zoom-in view of the three segment tapered cavity region, which is sandwiched between the top/bottom DBR with 25/30 mirror pairs, is included. In the central GaAs spacer, a single QD layer of InAs/GaAs with a low areal density of $\sim 2.2 \times 10^{9} \mathrm{~cm}^{-2}$ has been implemented. The sample design and processing routine are described in detail in Ref. 19. Fig. 1(a) shows the typical mode spectrum of a MP with $d_{c}=2.0 \mu \mathrm{m}$ for high excitation power $(2.5 \mathrm{~mW})$. The twofold polarization degeneracy of the fundamental mode is lifted by a slight ellipticity ${ }^{21}$ and two linearly polarized mode components $\mathrm{HE} 11_{\mathrm{X} / \mathrm{Y}}$ are emitting at $883.7 / 883.8 \mathrm{~nm}$, respectively. The complete spectrum is normalized to the absolute intensity of the HE11 $1_{X}$-mode, the dominating lasing mode. The next higher order modes are located at $877.1 \mathrm{~nm}$ (HE01), $878.1 \mathrm{~nm}$ (EH01), and $879.1 \mathrm{~nm}$ (HE21). For reasons of illustration, the spectrum of the higher order modes (red line) has been multiplied by a factor of 50, as higher order modes are strongly suppressed.

A detailed study of the $Q$ factor for different diameters was performed for adiabatic MPs of sample MP1. We determined the $Q$ factor of the bare cavity at pump powers sufficiently high to saturate the QD absorption at excitation powers close to threshold, i.e. prior to the onset of stimulated emission. The red stars in Fig. 1(b) show the results of this investigation. $Q$ factors close to 20.000 have been detected for large $d_{c} \sim 2 \mu \mathrm{m}$. When moving to smaller diameters, $Q$ is generally decreasing. This trend, however, is modulated with an oscillatory behavior, which we attribute to diameter dependent contributions to the reflection of the fundamental 

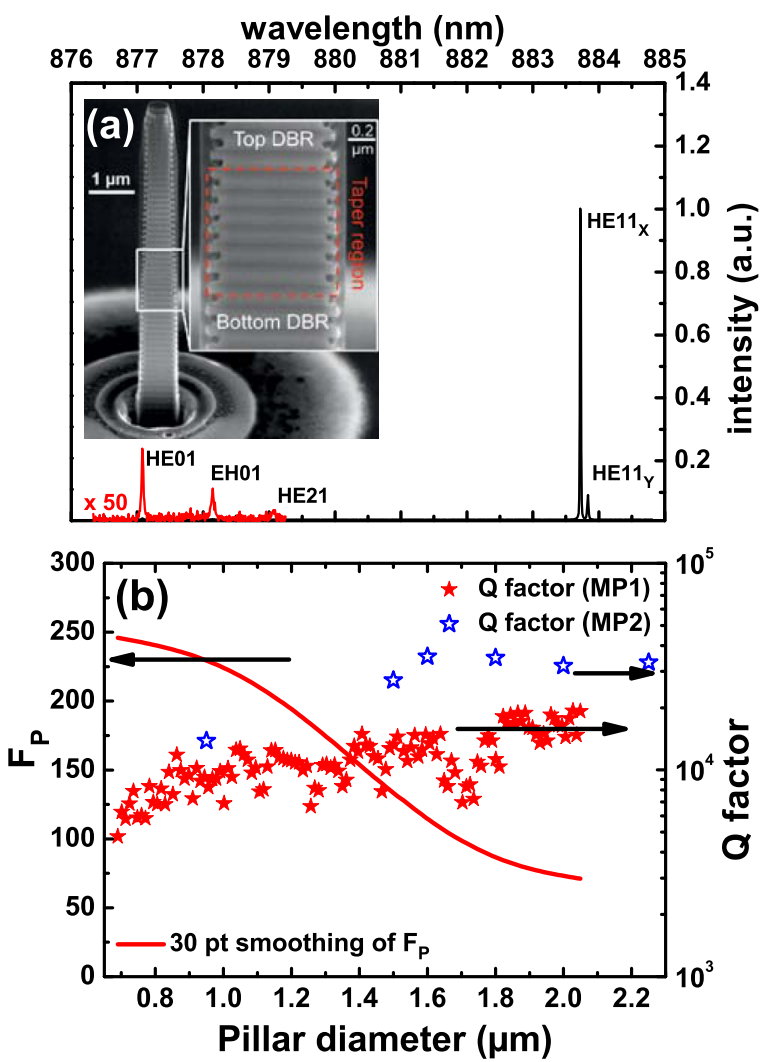

FIG. 1. (a) Typical mode spectrum of a micropillar (MP) with $d_{c}=2.0 \mu \mathrm{m}$ for high excitation power $(2.5 \mathrm{~mW})$. The twofold polarization degeneracy of the fundamental mode is lifted. Inset: SEM image of a vertically cut MP with $d_{c}=0.8 \mu \mathrm{m}$. (b) $Q$ factors of adiabatic MP cavities of sample MP1 (red stars) and the corresponding calculated maximum $F_{P}$ values with 30 point smoothing (solid red line). In addition, the $Q$ factors for the six MPs under investigation (sample MP2) are plotted with blue, open stars.

Bloch mode from higher order modes. ${ }^{11,22}$ While one would expect a drastic decrease of the $Q$ factor to values below 2.000 in the submicron range for standard MP cavities, ${ }^{20} Q$ factors around 10.000 have been measured in this diameter regime due to the positive influence of the adiabatic mode transition in the taper region. We thus observe a $Q$ factor reduction by only a factor of two if compared to $d_{c} \sim 2 \mu \mathrm{m}$, paving the way for the application of submicron MPs for suitable light sources exploiting cQED effects. This becomes even more obvious when taking $V_{\text {mode }}$ into account and calculating the expected maximal Purcell factor $F_{P}=3 / 4 \pi^{2}\left(\lambda_{c} / n\right)^{3} Q / V_{\text {mode }}$ of a single spatially and spectrally resonant QD in these MPs. In order to average over the $Q$ factor variations, a 30 point smoothing has been applied (solid red line) and is plotted in Fig. 1(b). A nominal value of $F_{P}=70$ is possible even for adiabatic MPs with diameters $d_{c} \sim 2 \mu \mathrm{m}$. As $V_{\text {mode }}$ is decreased, $F_{P}$ increases for smaller diameters, reaching values above 200 for $d_{c}<1.0 \mu \mathrm{m}$. Almost thresholdless lasing action should be feasible over the complete diameter range, which is described in terms of the $\beta$ factor and defined as the fraction of the SE coupled into the lasing mode. For MP cavities, it can be estimated to ${ }^{23}$

$$
\beta=F_{P}{ }^{*} /\left(F_{P}{ }^{*}+1\right) .
$$

For QD-ensemble lasing, as explored here, one has to average over the contributions of all QD lines in the ensemble, leading to an effective Purcell factor $F_{P}{ }^{*}<F_{P}$. Additionally, one has to take into account that the orientation of the dipole moment of the QDs is randomly distributed and the QDs are spatially and spectrally misaligned relative to the lasing mode. ${ }^{24}$ Hence, the resulting $\beta$ factor in Eq. (1) is reduced, if compared to the nominal $F_{P}$ of an ideal, single QD coupled resonantly to the cavity mode. For the MPs under investigation (inhomogeneous ensemble of monochromatic emitters, spectral location of the lasing mode close to the maximum of the QD-ensemble and lifting of the twofold polarization degeneracy of the mode), we estimate a reduction of the averaged Purcell factor $F_{P}{ }^{*}$ by roughly one order of magnitude. ${ }^{24}$ Nevertheless, these microlasers should still show low threshold lasing action with $\beta$ values up to 0.97 , taking an averaged $F_{P}{ }^{*}$ of all QDs into account.

In order to investigate lasing properties of our devices, a slightly different processing routine has been adopted for sample MP2: MPs with diameters ranging from $2.25 \mu \mathrm{m}$ down to $0.95 \mu \mathrm{m}$ have been planarized with benzocyclobutene (BCB). $\mathrm{BCB}$ works as a sealing for the sidewalls in order to avoid not only oxidation but also contamination with water during the lift off step of the residual etch mask, consisting of barium fluoride $\left(\mathrm{BaF}_{2}\right)$ and chromium $(\mathrm{Cr})$. The processed sample was mounted in a cryostat (temperatures down to $10 \mathrm{~K}$ ), allowing to perform micro-photoluminescence ( $\mu \mathrm{PL}$ ) spectroscopy of individual MPs. To ensure efficient pumping of the QDs and to avoid pumping related heating of the sample, a wetting layer excitation scheme $(\sim 840 \mathrm{~nm})$ has been applied by means of a tunable cw Ti:Sapphire laser. The laser beam was focused on the sample via a microscope objective (numerical aperture $=0.4$ ). The same objective was used to collect the emitted light, dispersed by a grating monochromator and detected by a $\mathrm{LN}_{2}$ cooled Si-CCD camera. An overall spectral resolution of about $16 \mu \mathrm{eV}$ could be achieved.

We systematically studied six MPs with various diameters $\left(d_{c}=2.25 \mu \mathrm{m}, 2.0 \mu \mathrm{m}, 1.8 \mu \mathrm{m}, 1.6 \mu \mathrm{m}, 1.5 \mu \mathrm{m}\right.$, and $0.95 \mu \mathrm{m}$ ) and observed strong evidence for lasing action of both parts of the fundamental mode $\left(\mathrm{HE} 11_{\mathrm{X}, \mathrm{Y}}\right)$ with very high $Q$ factors around $30.000\left(d_{c} \geq 1.5 \mu \mathrm{m}\right)$ and 14.000 $\left(d_{c}=0.95 \mu \mathrm{m}\right)$. In the following, we are focusing for each MP on the component of the fundamental mode with higher emission intensity. The open blue stars in Fig. 1(b) show the $Q$ factors of these microlasers. Table I gives a

TABLE I. Summary of results for the six MPs investigated, with diameters ranging from $2.25 \mu \mathrm{m}$ to $0.95 \mu \mathrm{m}$. From the measured $Q$ factors, the nominal Purcell factors $F_{P}$, the effective Purcell factors $F_{P}{ }^{*}$, the expected $\beta$ values, and the mean number of QDs in the MPs $\left(\#_{Q D S_{-} M P}\right)$ have been estimated. The measured $\beta$ values have been extracted with a simple REM and with a MicM. The fit parameters $\xi$ and $\#_{Q D S_{-} L M}$ of the MicM are added.

\begin{tabular}{lcccccc}
\hline \hline$d_{c}$ & $2.25 \mu \mathrm{m}$ & $2.0 \mu \mathrm{m}$ & $1.8 \mu \mathrm{m}$ & $1.6 \mu \mathrm{m}$ & $1.5 \mu \mathrm{m}$ & $0.95 \mu \mathrm{m}$ \\
\hline$Q$ & 27.400 & 32.100 & 36.000 & 36.000 & 26.700 & 14.000 \\
$F_{P}$ & 114 & 172 & 242 & 311 & 265 & 361 \\
$F_{P}{ }^{*}$ & 10 & 16 & 22 & 29 & 24 & 33 \\
$\beta$ & 0.91 & 0.94 & 0.96 & 0.97 & 0.96 & 0.97 \\
$\beta_{\text {REM }}$ & & & 0.33 & 0.47 & 0.44 & 0.61 \\
$\beta_{\text {MicM }}$ & & & 0.40 & 0.50 & 0.55 & 0.53 \\
$\xi$ & & 0.80 & 0.54 & 0.48 & 0.85 \\
\# $_{\text {QD_LM }}$ & & & 35 & 22 & 18 & 12 \\
\# $_{\text {QDS_MP }}$ & & & 56 & 45 & 39 & 16 \\
\hline \hline
\end{tabular}


compendium of the figure of merits of the six MPs, including $Q$, the expected nominal $F_{P}$, the effective $F_{P}{ }^{*}$ and $\beta$.

Fig. 2 presents a comprehensive study of both the inputoutput characteristics (black bullets) in double logarithmic presentation and the excitation power dependency of the linewidth $(\Delta \mathrm{E})$ (red squares) of the dominating lasing mode of the six MPs.

S-shaped behavior of the input-output characteristic is observed for the four smallest MPs, which reflects the smooth transition between the linear increase below threshold (marked with blue striped box) and the linear increase due to the dominant stimulated emission. Saturation of the output intensity occurs at high excitation powers, which is in agreement with theoretical predictions taking Pauli-blocking within a microscopic laser model into account. ${ }^{25}$ For $d_{c}=2.25 \mu \mathrm{m}$ and $2.0 \mu \mathrm{m}$, the dominant stimulated emission region is hardly reached. Interestingly, there is a significant decrease of the threshold pump power with decreasing $d_{c}$ which indicates higher $\beta$ factors for small diameter MPs as expected from Table I.

Another evidence for lasing action in the MPs is the excitation power dependence of the linewidth of the lasing mode. Whereas below threshold we observe only a small reduction of $\Delta \mathrm{E}$ due to saturation of material absorption by

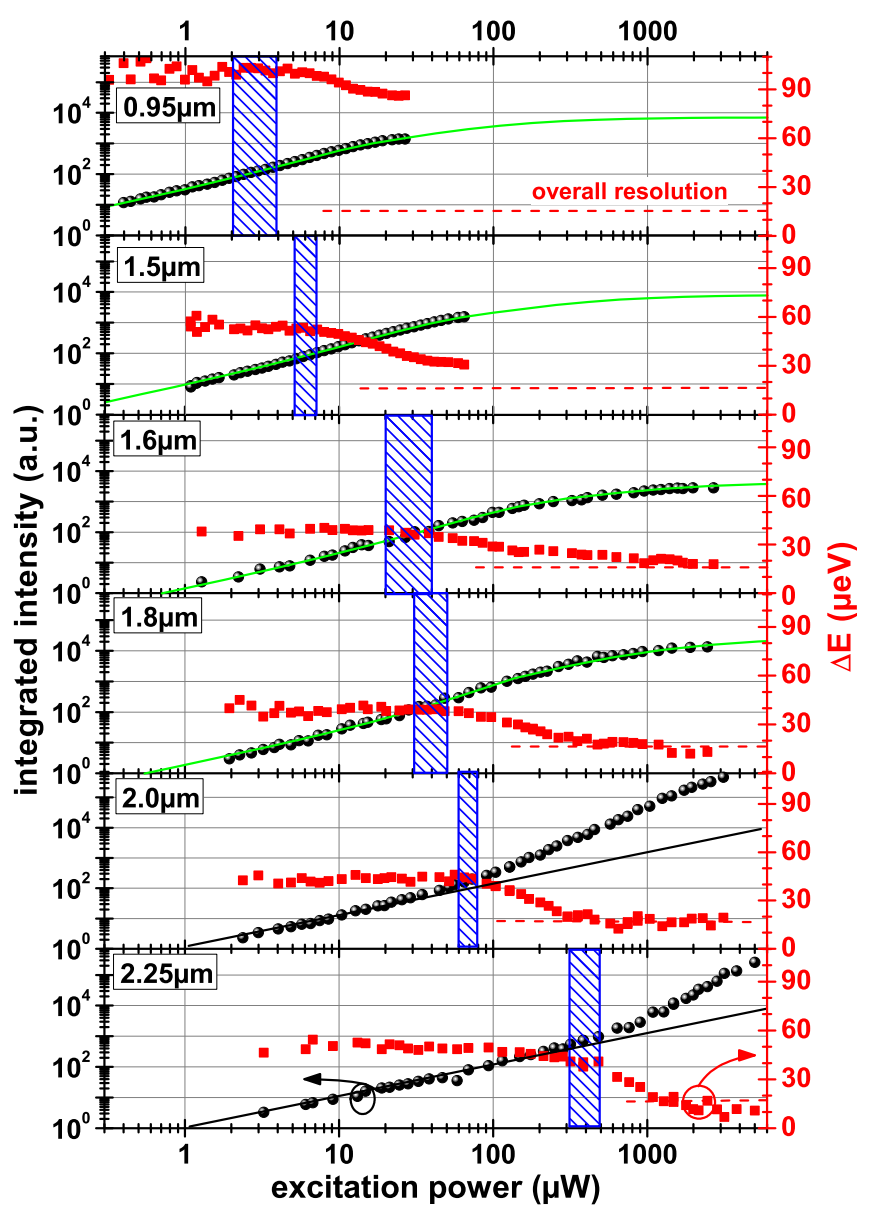

FIG. 2. Intensity (black bullets, left y-axis) and emission linewith $(\Delta \mathrm{E})$ (red squares, right y-axis) versus excitation power. The lasing threshold, marked with blue striped boxes, shifts monotonically from $400 \mu \mathrm{W}$ for $d_{c}=2.25 \mu \mathrm{m}$ to $3 \mu \mathrm{W}$ for $d_{c}=0.95 \mu \mathrm{m}$, while the s-shaped input-output characteristic gets less pronounced. Furthermore, the linewidth narrowing is less pronounced for smaller diameters. The solid green lines show the theoretical curves calculated with the MicM.
QDs, a strong decrease occurs with the onset of lasing action, indicating an increase of temporal coherence. ${ }^{13}$ In the lasing regime, a very narrow lasing line develops for MP-lasers with moderate $\beta$ values which typically cannot be resolved owing to the limited spectral resolution of the setup. The dashed red line in Fig. 2 marks the resolution limit of our setup. For the three MPs with the largest diameters $\left(d_{c}=2.25,2.0\right.$, and $\left.1.8 \mu \mathrm{m}\right), \Delta \mathrm{E}$ in the lasing regime is resolution limited and the transition from spontaneous emission to lasing action is given by a smooth kink close to the threshold. For the smaller MPs, this behavior is less pronounced and minimum lasing linewidths are determinable: $20 \mu \mathrm{eV}$ for $d_{c}=1.6 \mu \mathrm{m}, \quad 30 \mu \mathrm{eV}$ for $d_{c}=1.5 \mu \mathrm{m}$, and $85 \mu \mathrm{eV}$ for $d_{c}=0.95 \mu \mathrm{m}$. One can observe that the linewidth far in the lasing regime is increasing significantly with decreasing $d_{c}$, which comes along with a continuous increase of $F_{P}{ }^{*}$ and, due to Eq. (1), $\beta$. The $\beta$ dependence of the lasing linewidth has already been observed in high $\beta$ microdisc lasers and has been attributed to refractive index fluctuations due to nonequilibrium carrier generation above threshold. ${ }^{26}$ The MP with $d_{c}=0.95 \mu \mathrm{m}$ shows a linewidth narrowing of only $\sim 15 \%$ deep in the lasing regime, in good agreement with literature on high $\beta$ microlasers. ${ }^{16,27}$ Moreover, the smoother transition from SE to stimulated emission indicates higher $\beta$ values in the small diameter range.

As $\beta$ characterizes the threshold behavior of the microlaser, we find a consistent decrease from $\sim 400 \mu \mathrm{W}$ for $d_{c}=2.25 \mu \mathrm{m}$ to $\sim 3 \mu \mathrm{W}$ for $d_{c}=0.95 \mu \mathrm{m}$ of the threshold of the devices. This is in total a reduction by more than 2 orders of magnitude.

We applied two different modeling techniques to the s-shaped input-output characteristics of the four smallest MPs. The first approach is based on a microscopic model (MicM), introduced in Ref. 25. Here, the light-matter interaction is treated on a microscopic footing and carrier-photon and photon-photon correlations are included on the basis of the so-called cluster expansion technique. This method has been used to study input-output characteristics as well as photon statistics in QD based microcavity systems ${ }^{28}$ allowing to extract $\beta, \xi$ (mean number of SE photons in the cavity at the point of transparency) and the effective number of QDs emitting into the lasing mode $\left(\#_{\mathrm{QDs} \_ \text {LM }}\right)$. The second method is based on a simple rate equation model (REM) and is described in Ref. 29. In contrast to Ref. 29, we put $\beta$ as the only fit parameter of the REM, while we used $\xi$ from the MicM and measured the linewidth of the bare cavity. The fitted $\beta$ values, $\xi$ and $\#_{Q D S_{-} L M}$ are summarized in Table I. We observe very good consistency between the two models and the experimental data. The MicM also reproduces the saturation region for high excitation powers. In Fig. 2, we show the input-output curves, obtained from the MicM. As the input-output characteristic for $d_{c}=0.95 \mu \mathrm{m}$ is almost a flat line, the extracted $\beta$ has a large uncertainty. For $d_{c}=2.25 \mu \mathrm{m}$ and $2.0 \mu \mathrm{m}$, we hardly reach the dominant stimulated emission regime, which allows us to estimate only an upper boundary for $\beta$ of the order of 0.01 .

When comparing the calculated $\beta$ s with the fitted ones, we notice a large overestimation, especially in the large diameter range. We attribute this mainly to the fact that QDs are not emitting monochromatically but show a homogenous 
broadened lineshape for nonresonant excitation (typical $\sim 40 \mu \mathrm{eV}$ for low excitation). For high excitation, which is necessary to enter the stimulated emission regime for the large diameters, the Lorentzian shaped transition even changes into a broad background emission spaced out over some meV. ${ }^{30}$ This also points to the main limitation of the used microresonator system: Depending on the excitation power, the energy splitting of $\mathrm{HE} 11_{\mathrm{X}}$ and $\mathrm{HE} 11_{\mathrm{Y}}$ (50$100 \mu \mathrm{eV}$ ) is for all six MPs of the order or even much smaller than the homogenous linewidth of the QDs. Consequently, QDs can simultaneously be coupled to both lasing modes, which prevents thresholdless lasing action so far and could be circumvented by patterning MPs with a more elliptical cross-section.

It is instructive to consider on the number of QDs located in the active layer of the MPs $\left(\#_{Q D S_{-} M P}\right)$, which can be calculated using the areal QD-density of the sample. Compared with $\#_{Q D S_{-} L M}$ (cf. Table I), it turns out that roughly half of the QDs in the MPs are emitting effectively into the dominant lasing mode, indicating the limitation of our microresonator system.

We further expect the coupling to the collection optics being reduced for pillar diameters below $1.5 \mu \mathrm{m}$ due to a widening of the far field pattern. In this regime, the coupling can be optimized using tapering techniques similar to those used in photonic nanowires. ${ }^{31}$

In conclusion, adiabatic MP cavities form an advanced microresonator system, supporting high $Q$ factors above 10.000 both in the diameter range $\sim 2 \mu \mathrm{m}$ and submicron diameter regime. This allows us to perform a detailed study of low threshold lasing action for a variety of $\beta$ factors and the characteristics for high $\beta$ lasing applications such as linewidth narrowing and superlinear output increase. Even thresholdless lasing action in such a microresonator system might be possible, when addressing the limitation by elliptical MPs due to state of the art processing routines or spectroscopic measurement methods. Moreover, we observe very good consistency between two modeling techniques for input-output characteristics of microlasers, which gives us a substantiated insight into the reliability of both models and the horizon of expectations of the variables.

The authors thank A. Wolf and M. Emmerling for expert sample preparation. We are grateful to the State of Bavaria and Villum Fonden (NG and JM) for the financial support.

${ }^{1}$ T. Baba, T. Hamano, F. Koyama, and K. Iga, IEEE J. Quantum Electron. 27, 1347 (1991).

${ }^{2}$ J. M. Gérard, D. Barrier, J. Y. Marzin, R. Kuszelewicz, L. Manin, E. Costard, V. Thierry-Mieg, and T. Rivera, Appl. Phys. Lett. 69, 449 (1996).

${ }^{3}$ J. M. Gérard, B. Sermage, B. Gayral, B. Legrand, E. Costard, and V. Thierry-Mieg, Phys. Rev. Lett. 81, 1110 (1998).
${ }^{4}$ J. P. Reithmaier, G. Sek, A. Löffler, C. Hofmann, S. Kuhn, S. Reitzenstein, L. V. Keldysh, V. D. Kulakovskii, T. L. Reinecke, and A. Forchel, Nature 432, 197 (2004).

${ }^{5}$ E. Moreau, I. Robert, J. M. Gérard, I. Abram, L. Manin, and V. ThierryMieg, Appl. Phys. Lett. 79, 2865 (2001).

${ }^{6}$ M. Pelton, C. Santori, J. Vuckovic, B. Zhang, G. S. Solomon, J. Plant, and Y. Yamamoto, Phys. Rev. Lett. 89, 233602 (2002).

${ }^{7}$ T. Heindel, C. Schneider, M. Lermer, S. H. Kwon, T. Braun, S. Reitzenstein, S. Höfling, M. Kamp, and A. Forchel, Appl. Phys. Lett. 96, 011107 (2010).

${ }^{8}$ C. Santori, D. Fattal, J. Vuckovic, G. S. Solomon, and Y. Yamamoto, Nature 419, 594 (2002).

${ }^{9}$ A. Dousse, J. Suffczynski, A. Beveratos, O. Krebs, A. Lemaître, I. Sagnes, J. Bloch, P. Voisin, and P. Senellart, Nature 466, 217 (2010).

${ }^{10}$ J. M. Gérard, Top. Appl. Phys. 90, 269 (2003).

${ }^{11}$ S. Reitzenstein, N. Gregersen, C. Kistner, M. Strauss, C. Schneider, L. Pan, T. R. Nielsen, S. Höfling, J. Mork, and A. Forchel, Appl. Phys. Lett. 94, 061108 (2009).

${ }^{12}$ A. Dousse, J. Suffczynski, R. Braive, A. Miard, A. Lemaitre, I. Sagnes, L. Lanco, J. Bloch, P. Voisin, and P. Senellart, Appl. Phys. Lett. 94, 121102 (2009).

${ }^{13}$ S. Reitzenstein, A. Bazhenov, A. Gorbunov, C. Hofmann, S. Münch, A. Löffler, M. Kamp, J. P. Reithmaier, V. D. Kulakovskii, and A. Forchel, Appl. Phys. Lett. 89, 051107 (2006).

${ }^{14}$ S. Reitzenstein, T. Heindel, C. Kistner, A. Rahimi-Iman, C. Schneider, S. Höfling, and A. Forchel, Appl. Phys. Lett. 93, 061104 (2008).

${ }^{15}$ Z. G. Xie, S. Götzinger, W. Fang, H. Cao, and G. S. Solomon, Phys. Rev. Lett. 98, 117401 (2007).

${ }^{16}$ S. Reitzenstein, C. Böckler, A. Bazhenov, A. Gorbunov, A. Löffler, M. Kamp, V. D. Kulakovskii, and A. Forchel, Opt. Express 16, 4848 (2008).

${ }^{17}$ M. Nomura, N. Kumagai, S. Iwamoto, Y. Ota, and Y. Arakawa, Opt. Express 17, 15975 (2009).

${ }^{18}$ M. Khajavikhan, A. Simic, M. Katz, J. H. Lee, B. Slutsky, A. Mizrahi, V. Lomakin, and Y. Fainman, Nature 482, 204 (2012).

${ }^{19}$ M. Lermer, N. Gregersen, F. Dunzer, S. Reitzenstein, S. Höfling, J. Mørk, L. Worschech, M. Kamp, and A. Forchel, Phys. Rev. Lett. 108, 057402 (2012).

${ }^{20}$ G. Lecamp, J. P. Hugonin, P. Lalanne, R. Braive, S. Varoutsis, S. Laurent, A. Lemaitre, I. Sagnes, G. Patriarche, I. Robert-Philip, and I. Abram, Appl. Phys. Lett. 90, 091120 (2007).

${ }^{21}$ S. Reitzenstein, C. Hofmann, A. Gorbunov, M. Strauß, S. H. Kwon, C. Schneider, A. Löffler, S. Höfling, M. Kamp, and A. Forchel, Appl. Phys. Lett. 90, 251109 (2007).

${ }^{22}$ P. Lalanne, J. P. Hugonin, and J. M. Gérard, Appl. Phys. Lett. 84, 4726 (2004).

${ }^{23}$ W. L. Barnes, G. Björk, J. M. Gérard, P. Jonsson, J. Wasey, P. T. Worthing, and V. Zwiller, Eur. Phys. J. D 18, 197 (2002).

${ }^{24}$ B. Gayral, Ann. Phys. (France) 26, 1 (2001).

${ }^{25}$ C. Gies, J. Wiersig, M. Lorke, and F. Jahnke, Phys. Rev. A 75, 013803 (2007).

${ }^{26}$ U. Mohideen, R. E. Slusher, F. Jahnke, and S. W. Koch, Phys. Rev. Lett. 73, 1785 (1994).

${ }^{27}$ M. Nomura, Y. Ota, N. Kumagai, S. Iwamoto, and Y. Arakawa, Appl. Phys. Lett. 97, 191108 (2010).

${ }^{28}$ S. M. Ulrich, C. Gies, S. Ates, J. Wiersig, S. Reitzenstein, C. Hofmann, A. Löffler, A. Forchel, F. Jahnke, and P. Michler, Phys. Rev. Lett. 98, 043906 (2007).

${ }^{29}$ P. Jaffrennou, J. Claudon, M. Bazin, N. S. Malik, S. Reitzenstein, L. Worschech, M. Kamp, A. Forchel, and J. M. Gerard, Appl. Phys. Lett. 96, 071103 (2010).

${ }^{30}$ S. Strauf, K. Hennessy, M. T. Rakher, Y.-S. Choi, A. Badolato, L. C. Andreani, E. L. Hu, P. M. Petroff, and D. Bouwmeester, Phys. Rev. Lett. 96, 127404 (2006).

${ }^{31}$ N. Gregersen, T. R. Nielsen, J. Mørk, J. Claudon, and J. M. Gerard, Opt. Express 18, 21204 (2010). 